\title{
Un programme de recherche technologique en formation des adultes
}

Une approche enactive de l'activité humaine et l'accompagnement de son apprentissage/développement

\section{Marc Durand}

\section{OpenEdition}

\section{Journals}

Édition électronique

URL : https://journals.openedition.org/educationdidactique/373

DOI : 10.4000/educationdidactique.373

ISBN : 978-2-7535-1619-9

ISSN : 2111-4838

\section{Éditeur}

Presses universitaires de Rennes

\section{Édition imprimée}

Date de publication : 1 décembre 2008

Pagination : 97-121

ISBN : 978-2-7535-0790-6

ISSN : 1956-3485

\section{Référence électronique}

Marc Durand, « Un programme de recherche technologique en formation des adultes », Éducation et didactique [En ligne], 2-3 | Décembre 2008, mis en ligne le 01 décembre 2010, consulté le 23 août 2022. URL : http://journals.openedition.org/educationdidactique/373 ; DOI : https://doi.org/10.4000/ educationdidactique.373 


\title{
UN PROGRAMME DE RECHERCHE TECHNOLOGIQUE EN FORMATION DES ADULTES Une approche enactive de l'activité humaine et l'accompagnement de son apprentissage/développement ${ }^{1}$
}

\author{
Marc Durand, FPSE, Université de Genève, RIFT
}

\begin{abstract}
Résumé : Cet article présente une approche scientifique en formation des adultes portant sur l'activité humaine, qui articule deux programmes : l'un de recherche empirique consacré à l'analyse de l'activité dans différentes pratiques, l'autre de conception de situations visant à favoriser son apprentissage/développement. Il se centre sur le programme technologique et argumente l'idée d'une relation organique avec le programme de recherche empirique. Après une présentation du concept de programme de recherche en sciences sociales et son extension technologique, sont décrits le noyau de ces programmes centrés sur le postulat principal d'enaction et celui complémentaire de " conscience pré-réflexive », puis les principales hypothèses du cadre théorique sémio-logique selon lequel est approchée l'activité humaine (partie 1). Des concepts et des problèmes spécifiques au programme technologique de formation considérés comme critiques sont ensuite détaillés : principes de conception de situations formatives, rôle de l'analyse de l'activité, conception des « espaces d'actions encouragées », apprentissage/développement de dispositions individuelles à agir collectivement, empan d'activité encouragée (Partie 2). La conclusion met cette approche en perspective par rapport à des approches voisines et à la généalogie des programmes «cours d'action», à l'évolution des rapports entre pratiques sociales et formation, et entre science et technologie dans le champ de l'éducation.
\end{abstract}

Mots clés : conception, activité, enaction, programme, conscience pré-réflexive, technologie

Marc Durand

\section{Des programmes scientifiques et technologiques en éducation}

Cette partie présente: a) la notion de programme et sa spécification en sciences sociales en un programme portant sur l'activité humaine, b) son extension au domaine de la technologie et la conception d'artefacts visant à favoriser son apprentissage/ développement, c) le noyau de ces programmes constitué dans le cadre de notre approche de deux postulats « d'enaction » et de « conscience préréflexive », d) les principes de constitution d'observatoires et laboratoires pour l'analyse et la conception dans le cadre de projets de recherche et technologique, e) le " cadre théorique sémio-logique » qui spécifie les programmes et dans lesquels se déploient ces travaux de recherche empirique et de conception (cf. Theureau 2004, 2006).

\section{Des programmes de recherche empirique et technologique en formation}

Le concept de «programme scientifique » dû à Lakatos (1994) a montré sa pertinence en sciences sociales (Giard, 1994 par exemple). Un programme s'organise autour d'un noyau dur constitué d'un nombre restreint de postulats, qui détermine la définition des objets théoriques et objets d'étude, la nature des problèmes traités, les méthodes d'investigation... Ce noyau dur n'est pas la cible de tentatives de réfutations: il est isolé par une ceinture de protection constituée d'hypothèses auxiliaires qui elles, sont testées et éventuellement réfutées sans nécessairement déboucher sur une invalidation du noyau dur et donc du programme. La dynamique et la productivité scientifique d'un programme relèvent par conséquent d'une double heuristique: l'une négative qui concerne la protection du noyau dur, l'autre positive qui tente de réfuter des hypothèses auxiliaires.

Nous adoptons les critères de scientificité définis par Theureau (2006) complétant et explicitant les propositions de Lakatos: a) « littéralisation de l'empirique », b) «réfutabilité », et c) « une relation organique (ou de détermination réciproque) avec une technique ». Ce troisième critère est important parce qu'il implique un positionnement de la 
recherche par rapport à la technique et la technologie. Cette question a été abordée en profondeur par Theureau (2006) dans la perspective de programmes notamment d'anthropologie et d'ergonomie cognitives, c'est-à-dire dans une visée de conception de situations de travail ayant pour objectifs principaux la performance, santé et sécurité des individus, et s'organisant en un programme technologique dont il a précisé les composantes et les critères de validité. Ces critères sont: " efficacité technico-organisationnelle-culturelle », " relation organique avec une (ou plusieurs) science(s) empirique(s) », relation à des valeurs explicites (Theureau, sous presse).

S'agissant de recherche en formation des adultes, cette visée technologique concerne la conception d'artefacts destinés à favoriser l'apprentissage/développement, et s'organise en un programme technologique de formation. Ce programme se concrétise par des pratiques de conception ayant pour objet l'activité des individus et des collectifs, et relevant de problématiques de formation spécifiques que nous considérons comme aussi cruciales au plan épistémologique et aussi utiles au plan social - et donc d'égale dignité -, que les pratiques de recherche scientifique. L'affirmation d'une structuration en programme ainsi que celle d'une relation organique avec des programmes de recherche empirique implique de penser une relation non applicationniste entre recherche et conception. Ce programme technologique ne dérive pas de recherches qui seraient situées en amont: il correspond au développement de problématiques propres et récuse une conceptualisation de l'action en termes d'application de connaissances (d'origine scientifique ou non). Cette relation organique signifie aussi que le programme technologique, outre la résolution de problèmes pratiques, a une fonction de validation et d'invalidation des énoncés issus des programmes scientifiques, de contribution à la génération d'hypothèses, et contribue de façon plus large à fécondation. Pour résumer nous dirons que cette articulation spécifie un rapport de co-définition ou de détermination réciproque entre programmes scientifique et technologique, qui sont en lien de cohérence logique et fonctionnelle, et se fécondent mutuellement tout en étant dotés d'une autonomie relative. C'est à la définition et illustration de cette approche, avec une centration sur le programme technologique et ses relations organiques avec le programme de recherche empirique qu'est consacré cet article.

\section{Les postulats "d'enaction" et de "conscience pré-réflexive "}

Ces programmes prennent et spécifient « l'activité humaine » comme objets d'étude et de conception. Cette spécification est tenue par une exigence de pertinence écologique et de rigueur scientifique. Elle est notamment contrainte par la visée de formation qui impose la définition d'objets théoriques, et d'objets d'étude et de conception, compatibles avec la pratique de formation, définie comme la conception et la mise en place d'environnements sollicitant potentiellement chez les individus et les collectifs engagés dans des dispositifs ad hoc une activité prometteuse en termes d'apprentissage/développement. Par apprentissage nous entendons l'acquisition, par les individus et les collectifs qu'ils constituent, de modes d'action nouveaux pour eux, issus du patrimoine culturel (notamment professionnel) en relation ou non avec un projet intentionnel de transmission de ces modes d'action par une instance de formation; par développement nous entendons la transformation du répertoire d'actions des individus et des collectifs qu'ils constituent, en relation ou non avec un projet intentionnel d'accompagnement par une instance de formation.

La notion d'activité est une réduction de la pratique sociale dans laquelle sont engagés les individus. La puissance explicative et opérationnelle des recherches dépend du degré et de la pertinence de la réduction de cette pratique à laquelle aboutit la définition de l'objet théorique. Le dilemme est de conserver autant que possible le caractère complexe, pluridimensionnel, de totalité signifiante... de la pratique, tout en la réduisant afin de la rendre accessible par les démarches, méthodes et concepts scientifiques. Dans l'approche présentée ici, l'activité est réduite par la définition d'objets théoriques divers, notamment: cours d'action, cours d'expérience, cours de vie relatif à une pratique (cf. p. 103). Ces objets théoriques sont tenus par les postulats d'enaction et de conscience pré-réflexive qui spécifient la notion d'activité humaine.

Le postulat d'enaction est issu des sciences de la vie et de la cognition; il dérive de celui d'autopoièse. À la différence des machines cybernétiques fabriquées par l'homme et qui produisent des actions ou des objets, les systèmes vivants ont la particula- 
rité de produire aussi leur propre organisation. Leur existence et organisation ne sont pas établies par ailleurs et préalablement à leur activité, mais tiennent à leur fonctionnement même: ils s'auto-produisent. L'autopoïèse désigne cette organisation circulaire ou circularité auto-référentielle. Pour Maturana \& Varela (1994), la capacité des systèmes vivants à engendrer et spécifier leur organisation dans leurs rapports avec l'environnement, par un processus incessant de remplacement de leurs composants résultant de leur propre pulsation de vie et compenser ainsi les perturbations provenant de cet environnement, constitue une propriété fondamentale dont la prise en compte doit guider l'approche scientifique du vivant. Le maintien de l'organisation du système ne signifie pas une absence de transformations de ce système: à chaque instant la dynamique de cette circularité auto-référentielle s'accompagne de transformations non de l'organisation (qui est invariante), mais de la structure du système (c'est-à-dire de ses formes successives liées aux modifications du réseau des relations entre ses éléments constitutifs).

Les rapports dynamiques d'un système à son environnement observés à travers les transformations de sa structure constituent son « couplage structurel »; ce couplage construit et modifie à chaque instant la structure du système dont, dans le même temps, il dépend. Ce couplage est asymétrique dans la mesure où c'est le système qui définit ce qui, de son environnement, le perturbe, c'est-à-dire ce qui est « pertinent ou significatif pour lui ». Il crée une perspective celle du système -, qui change à chaque instant en fonction de sa propre dynamique et des perturbations émanant de l'environnement. Un système vivant est relativement stable parce que sa source de variation fondamentale (et donc d'invariance) est sa propre organisation, c'est-à-dire le réseau des relations qui le définissent; tant que ces relations sont viables (tant qu'elles régénèrent les réseaux qui les ont produits) il y a stabilisation du système. Il convient donc de distinguer l'adaptation du système qui concerne son autopoïèse et constitue un invariant si le système est vivant, de sa viabilité qui concerne son couplage structurel et sa structure, qui peut être plus ou moins changeante et satisfaire des critères secondaires par rapport à l'autopoïèse (Brocklesby \& Mingers, 2005 ; Tsuchiya, 2006). Dans une perspective de formation, des critères de viabilité variés sont sélectionnés qui, s'ils sont adoptés par les formés, servent de leviers pour des transformations orientées de leur couplage structurel, c'est-à-dire des processus d'apprentissage/ développement: par exemple la précision d'un geste d'incision dans une lame de bois, la perception de l'état de la configuration de la pièce usinée sur une chaîne de montage automatisée, la compréhension de la difficulté rencontrée par un élève dans l'apprentissage d'un élément du programme...

Nous traduisons ces idées en considérant que chaque individu est un acteur structurellement couplé avec son environnement. À chaque instant le monde pour cet acteur et l'acteur lui-même sont «fait-émerger » ou "enactés ». Le concept « d'enaction » désigne cette émergence auto-référentielle en même temps que le caractère de totalité et de totalisation de l'activité; et l'asymétrie du couplage structurel est concrétisée par le fait que chaque acteur - en tant que système vivant - a un point de vue propre sur son environnement. Ce couplage structurel se transforme en permanence et certaines de ces transformations ont un caractère pérenne, au sens où elles correspondent à la reproduction de comportements par l'acteur.

Le postulat de conscience pré-réflexive caractérise l'idée que l'activité humaine s'accompagne d'un vécu donnant lieu pour une part à une expérience, qui correspond à ce que l'on désigne habituellement par conscience (Theureau, 2004, 2006). L'expérience d'une activité représente non l'existence d'un fait mais l'intuition d'une action (par exemple la conscience d'écrire). La notion d'expérience est précisée avec le concept de « conscience pré-réflexive » qui caractérise une modalité particulière de vécu, constitutive de l'activité. Il s'agit de la familiarité de l'acteur à lui-même et sa présence à soi continue accompagnant le flux de son activité (l'expérience d'être en train d'écrire ne constitue pas une action nouvelle distincte de celle d'écrire). Cette présence de l'acteur à son activité est l'expérience partielle de son couplage structurel, que Theureau (2006), caractérise comme son « effet de surface». Cette notion signifie qu'il ne s'agit ni d'un effet dans l'environnement (ou effet extrinsèque distinct de l'activité), ni d'une activité autre (la conscience pré-réflexive étant consubstantielle à l'activité) comme dans le cas d'une prise de conscience qui suppose un arrêt de l'action en cours et une nouvelle action prenant la première comme objet, ainsi qu'une re-construction au plan 
de la représentation des mécanismes intimes de l'action première (Piaget, 1974). La prise de conscience est une action délimitable et repérable dans le continuum d'activité de l'acteur qui s'accompagne potentiellement d'une conscience pré-réflexive (faire l'expérience que l'on est en train de prendre conscience d'une action passée).

En résumé, la conscience pré-réflexive est considérée comme une composante de l'activité qui est exprimable (dans les conditions favorables présentées dans le § 1.3), continue (l'acteur y accède potentiellement à chaque instant), concomitante (elle n'interrompt pas le flux de l'activité) et partielle (toute l'activité ne fait pas expérience). Moyennant certaines conditions favorables, l'expression par l'acteur de cette expérience permet à l'observateur ou au formateur un accès à la dynamique interne et à la signification pour lui de son couplage structurel. Il s'agit d'un accès partiel puisqu'il ouvre seulement sur la part de l'activité qui est montrable, mimable, racontable et commentable par lui à un observateur engagé avec lui dans une enquête sur son activité.

\section{Observatoire et laboratoire pour la recherche et la conception}

Ces postulats d'enaction et de conscience préréflexive contraignent les modalités de recherche empirique et de conception des environnements de formation. Ces contraintes peuvent être énoncées sous forme de principes éthiques, ontologiques et épistémologiques (Theureau, 2006, sous presse) que nous résumons ici.

La recherche empirique vise à rendre compte de la dynamique du couplage structurel d'un acteur dans un environnement particulier, en respectant son asymétrie, c'est-à-dire en accordant dans la description un primat à son point de vue et en l'articulant avec celui d'un observateur (Theureau, 2004, 2006; Varela \& Shear, 1999). Cette phénoménologie empirique, a pour objet l'activité de l'acteur engagé dans une pratique professionnelle, artistique, familiale, de loisir..., conceptualisée comme une totalité dynamique, structurée et signifiante. Cela confère à l'analyse son orientation: il s'agit d'accéder à la conscience pré-réflexive de l'acteur et à la structuration située de son activité d'une part; de repérer et décrire les composantes de l'activité ne « faisant pas expérience » ainsi que les déterminants et effets extrinsèques du couplage structurel tels que la culture, la tâche et l'état de l'acteur d'autre part. Cela implique de rompre avec le décours naturel de l'activité et suppose une coopération particulière de l'acteur avec l'analyste. Dans sa forme usuelle, des traces de son activité sont enregistrées (généralement en vidéo) auxquelles il est ensuite confronté, afin de les mimer, montrer, commenter et raconter au cours d'une enquête avec l'analyste. L'hypothèse qui sous-tend ces séances dites d'auto-confrontation, est qu'en se confrontant à l'instant $t+1$ aux traces de son activité enregistrées à l'instant $t$, l'acteur accède à la conscience pré-réflexive accompagnant son activité à l'instant $t$, par une remise en situation dynamique. Cette remise en situation dynamique implique notamment un processus « d'immersion mimétique » à partir des ancrages que constituent les traces enregistrées de son activité (Schaeffer, 1999).

L'analyse procède par une déconstruction et reconstitution de la dynamique d'engendrement de l'activité. Elle aboutit à une description de sa structuration et de sa signification, qui procède par des va-et-vient entre le niveau local (un découpage en unités les plus réduites possibles mais significatives pour l'acteur) et le niveau global (les structures repérables à partir de l'identification des relations entre les unités élémentaires d'activité). L'analyse tend à dépasser la description des occurrences singulières de l'activité dans son environnement, pour atteindre des degrés plus ou moins élevés de généralisation, c'est-à-dire une typicalisation de cette activité. Ceci requiert de la part de l'analyste de spécifier une ou des occurrences comme étant une ou des occurrences types, c'est-à-dire condensant les traits partagés par les autres occurrences qui, en raison de ce partage sont considérées comme "membres de la famille des occurrences observées ». Être membre d'une famille ne signifie pas appartenir à une catégorie: certaines occurrences sont plus typiques que d'autres parce qu'elles possèdent davantage des traits caractéristiques et sont de meilleurs représentants de la famille que d'autres. Ces « familles » peuvent varier en extension: la famille des actions d'un acteur au travail, des actions de plusieurs acteurs dans le même travail, dans des pratiques professionnelles voisines, etc. Pour identifier le caractère typique d'une occurrence, deux indices sont retenus: l'expression par l'acteur 
de ce caractère de typicité lors des séances d'autoconfrontation, la validation a posteriori par lui des regroupements d'occurrences réalisées par l'analyste. Ceci permet secondairement de qualifier une occurrence observée comme étant typique, parce qu'elle condense le mieux les traits de cette famille.

Les acteurs engagés dans les protocoles de recherche tirent potentiellement de cette implication une aide à la performance et à l'apprentissage/développement. Le fait de rendre disponible pour chacun d'eux, le matériau empirique (enregistrements, verbatim des entretiens, descriptions et analyses par le formateur...) est susceptible d'enrichir leur environnement habituel et leur culture, et de déclencher une activité individuelle ou collective portant sur elle. Cet effet de formation a été peu étudié à ce jour. Mais il nous semble ressortir de la nature de l'activité induite chez les acteurs pendant l'enquête sur leur activité: observation et confrontation aux traces de leur activité ou celle d'autres acteurs, réponses aux questions de l'analyste, consultation des résultats, analyse critique de ces résultats et de leur interprétation...

La « suspension du jugement » qui est la condition d'une expression de leur conscience pré-réflexive précise et contrôlée, implique l'inhibition d'une activité évaluative spontanée et syncrétique et une focalisation sur l'activité réelle en dehors de tout jugement normatif. Cela rend possible un travail personnel et intime subséquent sur ces expériences. Les séances d'auto-confrontation impliquent une forme d'ascèse dans l'accès et l'expression contrôlée de la conscience pré-réflexive, ayant potentiellement un effet d'entraînement à cet exercice, et susceptible d'être reproduit en dehors de la recherche. L'évaluation critique, qui se distingue du jugement spontané, concerne l'activité réellement déployée et repose sur une mise en débat intime à partir de critères explicites d'efficacité pratique; elle aboutit à un renforcement des modes d'actions usuels, un ajustement de ces actions ou une recherche d'alternatives.

Par ailleurs, les «prises de conscience » qui peuvent accompagner la consultation des résultats et les analyses produites par le chercheur, même si elles ne sont pas expressément recherchées dans l'enquête, parce qu'elles donnent accès aux mécanismes intimes de l'action et sont des reconstructions au plan symbo- lique, aboutissent à des synthèses, généralisations et ouvertures de nouveaux possibles permettant un gain d'efficacité en lien avec les opportunités ainsi acquises de planification de ces actions et de transfert à des situations ou actions analogues. Enfin, la collaboration à la recherche peut avoir des effets généraux tels l'appel à l'imagination productrice, c'est-à-dire l'invention de moyens d'action alternatifs par ouverture de nouveaux possibles et combinaisons innovantes, et l'appropriation des concepts et notions théoriques qui peuvent s'accompagner d'un gain d'autonomie et de lucidité.

Il serait fallacieux de séparer radicalement l'analyse de l'activité d'un acteur de sa transformation liée à la procédure de recherche. C'est d'ailleurs pourquoi une approche scientifique qui porte sur l'activité humaine ne peut adopter sans discussion les distinctions issues des sciences exactes entre, par exemple éthique et épistémologie, science et technique, etc. C'est aussi pourquoi nous tentons de concevoir conjointement les rapports entre science et technologie dans notre approche de la formation des adultes. Il demeure cependant qu'une collaboration précise et contractuelle entre l'analyste/formateur et les acteurs est également requise dans le cadre du programme technologique. Dans ce cadre-ci, l'activité des acteurs fait a priori et fondamentalement l'objet d'une « mise au travail », qui suppose la réalisation d'un « laboratoire ». Le principe de cette mise au travail est de transformer la structuration et la signification de l'activité pour les acteurs/formés dans une orientation consensuellement définie par le formateur et les formés. À cette fin, des environnements d'apprentissage/développement sont conçus et mis à disposition des individus et des collectifs en formation.

Ceci implique une coopération entre formateur et formés cohérente avec les présupposés théoriques. En ce qui concerne le programme technologique, cette coopération a pour principal objectif la conception de dispositifs de formation. Cette activité de conception est dynamique et évolutive; elle a pu être qualifiée par ailleurs "d'ingénierie de contexte », « d'ingénierie concourante ou simultanée » ou « d'ingénierie en référence au fonctionnement des systèmes vivants » (Leblanc, Ria, Dieumegard, \& Durand, 2008; Le Boterf, 2003). La conception suppose de connaître l'activité susceptible d'être réalisée dans l'environnement conçu par le formateur, avant qu'elle ne le 
soit (Pinsky, 1992; Theureau \& Jeffroy, 1994). Elle commence donc par une sorte de pari sur la nature de l'activité, qui est la clé du processus de conception et sollicite la créativité du concepteur et sa connaissance de l'activité cible. Cette connaissance est issue de sa propre expérience de formateur, de la culture partagée de la pratique en question, et surtout des travaux de recherche portant sur la formation et l'analyse de l'activité. Une fois mise en œuvre, la formation suppose une démarche itérative alternant des phases de conception, d'analyse de l'activité des formés, d'ajustements de l'environnement, d'analyse de l'activité des formés, etc. Cela demande une coopération à long terme avec les formés qui: a) s'engagent en toute connaissance dans le projet technologique, b) consentent qu'on les observe lorsqu'ils sont engagés dans cet environnement formatif, c) expriment sincèrement leur expérience à cette occasion, d) contribuent à la conception par une évaluation guidée et des propositions de transformation de l'environnement, et parfois c) acceptent une délégation de la conception d'environnements de formation de $2^{\mathrm{e}}$ ou $3^{\mathrm{e}}$ génération conduisant à une auto-formation, à partir de principes préalablement précisés dans les phases initiales du projet.

\section{Le cadre théorique sémio-logique}

Les postulats de l'enaction et de la conscience pré-réflexive sont spécifiés par un corps d'hypothèses définissant un cadre théorique dit " sémio-logique » dont sont énoncés ici les traits principaux (Theureau, 2004, 2006, sous presse, pour des présentations approfondies).

L'activité est une permanence dynamique, un flux, que Theureau caractérise comme « ouvert aux deux bouts », c'est-à-dire non borné a priori, qui hérite à chaque instant de l'histoire de l'activité passée, est ouvert sur un futur indéterminé et s'autoorganise. Ce décours d'activité est ordonné sans être réductible à une prescription par des préalables cognitifs ou culturels. Il résulte d'états successifs du couplage structurel qui sont analysés dans la perspective de reconstruire ou d'infléchir leur dynamique d'émergence.

C'est donc considérer l'activité comme située, ou comme couplée à un environnement particulier, mais comme ne s'y adaptant pas dans la mesure où elle spécifie ce qui, de cet environnement, la perturbe, et se spécifie elle-même dans ce couplage. L'activité fait émerger des « micro-identités » et des « micromondes'(Varela, 1989, 1996) qui sont « enactés », ou «fait émerger » à chaque instant. Le concept de situation prend un sens particulier dans ce cadre: il ne s'agit pas d'un artefact face auquel l'acteur serait posé, mais d'un processus de spécification. La situation ou monde propre exprime le point de vue de l'acteur sur son environnement. Ce point de vue est global, ce qui autorise à énoncer que l'activité est tout à fois corporellement, spatialement, temporellement, culturellement... située.

Ce cadre théorique ne pose pas un sujet d'emblée structuré face à un objet pré-donné : il situe les sources de permanence et de régularité non dans le sujet et l'objet mais dans le couplage structurel (et autopoiétique) ou l'activité qui les unit tout en les spécifiant. C'est dire que les notions de subjectivité et d'objectivité ont un sens particulier: le «monde propre » du système n'a pas une identité définitive et permanente puisque le « moi ou corps propre » fluctue selon l'activité en cours (la raquette du joueur de tennis est intégrée dans son corps propre lorsqu'il joue: une incorporation ou appropriation s'est produite et l'objet-raquette est devenu « transparent » pour lui; il peut agir sur la balle et obtenir des sensations en cours de jeu sans que cet objet fasse partie de sa conscience pré-réflexive; mais cette raquette ne fait plus partie de son corps propre lorsqu'il réalise une autre activité ou exerce une activité «sur elle » telle que réparer son cordage). La limite entre moi propre/corps propre et monde propre est enactée; elle définit des microidentités transitoires en fonction des circonstances et des micro-mondes changeants. L'enaction recouvre des processus complémentaires d'individuation ou d'émergence d'une identité du système, et d'appropriation ou d'émergence d'un monde agi ou « agissable » (Simondon, 1989; Varela, 1996).

L'acteur définissant son monde propre à partir des éléments de l'environnement qui le perturbent, ces éléments sont pertinents pour lui c'est-à-dire significatifs (Von Uexküll, 1965; Varela, 1989). L'activité est donc une construction continue de signification; ce qui est résumé par la formule de Peirce complétée par Theureau (2004) «l'homme pense et agit par signes », c'est-à-dire déploie une « activité-signe ». 
Ces signes sont des outils ou médiateurs sémiotiques qui contribuent à la structuration de l'activité, à son ancrage culturel et sa transmission, et à l'articulation collective des activités individuelles.

Le flux d'activité peut être analysé comme une concaténation et un enchâssement d'unités discrètes d'activité qui sont des signes ou des unités de signification. Celles-ci se regroupent selon une pragmatique et entretiennent entre elles des relations de dépendance; elles réalisent ainsi des emboîtements ou enchâssements d'unités élémentaires d'action et/ou de signification. Ces structures émergentes expriment un ordre (généralement accessible à un observateur familier avec la pratique en question). Cet ordre et cette signification peuvent être anticipés ou planifiés par l'acteur, cependant ces plans n'ont pas une fonction de programmation prescrivant l'accomplissement situé, mais une ressource disponible dans l'action (Suchman, 1987).

Certains états du couplage donnent lieu à une généralisation: parce qu'elles se répètent ou parce qu'elles sont particulièrement pertinentes et significatives, certaines occurrences sont typicalisées et deviennent des types. Ce processus de typicalisation consiste en l'extension de la signification d'une occurrence, qui prend une valeur d'ancrage pour les expériences passées, présentes et futures. Si, pour reprendre les catégories de Peirce, les occurrences sont du registre du factuel ou de la secondéité, les types eux sont de l'ordre du général, de la règle, du savoir, du virtuel, de la tiercéité. Ils peuvent une fois construits, être actualisés dans des circonstances autres que celles où ils ont été construits et ayant pour l'acteur un air de famille. L'actualisation d'un type peut contribuer à son renforcement ou son affaiblissement. Ces types constituent la culture de l'acteur et, lorsqu'ils sont partagés par différents acteurs, ils constituent une culture commune.

Les occurrences sont des actualisations de possibles: chaque action réalise un tri entre possibles actualisés et non actualisés, et opère donc une césure avec ce qui n'a pas été actualisé. Ces possibles relèvent d'un registre d'expérience particulier: celui de la priméité (Peirce, 1978). L'activité est héritière de l'activité passée et son actualisation ici et maintenant débouche virtuellement sur une généralisation: à chaque instant une typicalisation est possible ouvrant sur le registre de la tiercéité. C'est dans ce passage entre possible, actuel et virtuel que s'originent des transformations plus ou moins pérennes de l'activité. Chaque action constitutive du flux d'activité émerge à chaque instant d'une indétermination initiale sous forme a) d'ouverture de possibles délimitant tout ce qui pourrait advenir à l'instant $t$ (en fonction du projet de l'acteur, de ses dispositions à agir issues de sa culture, de l'histoire en cours, de l'état de l'environnement...), b) d'une actualisation sous forme d'accomplissement situé de certains de ces possibles, et c) d'une typicalisation de certaines de ces occurrences.

Dans ce cadre théorique sémio-logique, différents objets sont définis (Theureau, 2006), dont nous retenons dans cet article: le " cours d'expérience » qui désigne le flux d'activité faisant expérience pour un acteur dans un contexte donné (la signification que prend pour un voyageur son trajet dans le métro), le " cours d'action » individuel ou collectif qui désigne les relations entre le cours d'expérience et ses déterminants et effets extrinsèques: culture, tâche, état de l'acteur (les transformations de l'activité d'un individu engagé dans une situation de formation en fonction des modifications de son environnement d'apprentissage) et le « cours de vie relatif à une pratique », qui porte sur une activité étendue dans le temps généralement discontinue (l'activité distribuée sur plusieurs mois ou années, d'un débutant qui alterne des périodes de formation formelle en institut ou sur le terrain, des pratiques en responsabilité, et des épisodes informels en lien avec son travail et son apprentissage/développement).

\section{Contribution à un programme technologique de formation}

Ce programme technologique est en relation organique avec des programmes de recherches empiriques (et notamment notre propre programme visant une phénoménologie de l'activité et des formations dans des pratiques sociales variées) qu'il contribue à dynamiser et valider, et dont il exploite les avancées. Nous parlons de programme technologique plutôt que « d'ingénierie de formation » ou de « didactique » afin de marquer divers points de différence avec ce qu'on dénomme ainsi en éducation. 
Il s'agit d'un programme technologique et non d'une ingénierie dans la mesure où il ne consiste pas en la réalisation quotidienne de formations, en exploitant des outils et moyens disponibles ou appliquant des connaissances scientifiques à des fins pratiques, mais en la conception d'outils innovants pour agir dans des environnements dont la problématique est porteuse de questions clés d'apprentissage/développement individuel et collectif (Durand, sous presse a). Ce programme est un ensemble organisé de valeurs, pratiques et conceptualisations tenant leur cohérence de postulats en nombres limités, qui vise une production d'artefacts conséquente avec ces postulats, dont les processus de production sont rendus les plus explicites possibles, dont l'efficacité en termes de formation est évaluée rigoureusement, et qui sont traduits en processus et produits typiques dans la perspective de dépasser les cas particuliers traités dans le cadre de projets spécifiques. Ces projets technologiques sont des réponses à des demandes émanant d'acteurs confrontés à des problèmes pratiques, adressées à notre équipe.

Bien qu'originellement inspiré par la didactique professionnelle (par exemple Pastré, 2004), et orienté vers des questions de transposition didactique, il ne s'agit pas d'un programme de didactique dans la mesure où la question des objets d'enseignement et de formation n'est pas la seule traitée. En raison de sa dynamique propre ce programme a abouti à une ouverture sur des problématiques plus larges (Durand, sous presse b). Enfin l'activité est appréhendée comme une totalité en tentant de respecter autant que possible son intégrité; nous n'adoptons pas le découpage a priori, fréquent dans les recherches en enseignement scolaire, entre pédagogie et didactique, parce qu'il ne permet pas un accès aux processus de structuration et signification globales de l'activité.

Cette partie présente les principes, concepts et problèmes critiques de conception de formation sous les présupposés, méthodes et cadre théoriques présentés dans la première partie.

\section{Principes de conception de formation centrée sur l'activité}

Ce programme technologique exploite partiellement et dans une perspective particulière les principes de la « conception centrée sur le cours d'action » en ergonomie (Pinsky, 1992; Theureau \& Jeffroy, 1994), dont les principaux sont listés ci-dessous.

Le principe d'analyse critique et de traduction des demandes de formation. Ce principe organise les préalables et les contenus des projets de formation. Ces demandes émanent d'acteurs engagés dans des pratiques familiales, artistiques, de travail, de sport de haut niveau, de loisir, de formation, etc ${ }^{2}$. Cette demande reçoit une réponse positive d'engagement dans un projet technologique sous réserve d'une analyse critique (qui peut être approfondie et nécessiter une enquête de terrain) lorsque trois conditions sont réunies (outre la capacité des concepteurs à répondre et conduire le projet): a) le caractère inédit, actuel et significatif des problématiques de formation posées - et plus ou moins reconstruites selon le cas -, à partir de la demande initiale (par exemple la demande d'accompagnement de danseurs professionnels en fin de carrière peut être considérée comme condensant des problèmes d'actualité en matière de formation des adultes: conception de dispositifs d'accompagnement, formation tout au long de la vie, validation d'acquis de l'expérience, capitalisation de compétences et réinsertion, etc.) ; b) la relation étroite entre ces problèmes de formation et des questions théoriques pertinentes pour notre programme de recherche empirique (par exemple les fins de carrière des danseurs posent des problèmes théoriques variés et complexes de redéploiement de dispositions à agir, de conceptualisation des parcours biographiques en termes de phases et transitions de phases, de spécificité et généralité des apprentissages, d'analyse et conceptualisation de l'activité à des empans temporels longs, d'unité/clivage identitaire, etc.) ; c) l'adhésion des acteurs de terrain à une dynamique d'intervention et une coopération qui est toujours réalisée dans la durée, et s'accompagne de transformations et mises en question des pratiques usuelles.

Le principe de conception de situations. Si l'activité du concepteur aboutit à la réalisation d'artefacts, de fait ce sont les situations (au sens précisé p. 102) qui constituent sa perspective: les artefacts représentent des offres ou des possibles pour les acteurs, dont l'actualisation en termes d'action située dépend d'eux. Autrement dit, le concepteur propose 
des artefacts que les acteurs ou formés intègrent ou non comme éléments significatifs dans leur couplage structurel en relation avec sa dynamique et signification propres (qui recoupe à divers degrés et sur un mode non prescrit, celle du concepteur). Ces artefacts transforment l'environnement des acteurs et perturbent potentiellement leur activité. Ce qui est visé par le concepteur est donc le couplage structurel des formés dans les environnements transformés par l'insertion de ces artefacts qui constituent des « appels', et servent d'ancrage potentiel pour l'activité souhaitée par eux (Theureau, sous presse). Insérés dans ces environnements ces artefacts sont censés perturber l'activité spontanée des formés, infléchir leur cours d'action et favoriser l'apparition de nouvelles dispositions, par typicalisation et appel à leur imagination et leur créativité. En raison des propriétés d'ouverture et d'indétermination de l'activité des formés, le concepteur ne possède jamais de certitude a priori quant à l'atteinte des effets attendus.

Le principe de dépassement. Ce principe peut être formulé sur un mode paradoxal: «faire faire aux acteurs ce qu'ils ne savent pas faire $»$. Sans ce dépassement, les propositions du concepteur ne sont que des occasions de reproduction, dans lesquelles les acteurs réalisent du déjà connu, agi, perçu, éprouvé... Les situations sont formatrices si elles diffèrent des situations cibles (notamment de travail) sans quoi il suffirait par exemple d'agir pour maîtriser ces pratiques, mais appellent en même temps des couplages différents des couplages actuels des formés: elles doivent les inciter à'monter sur leurs propres épaules », et à affronter ce qui est pour eux de "l'inagi » (c'està-dire du partiellement inconnu, «inéprouvé », «imperçu », inattendu, etc.). Les concepteurs sont là confrontés aux problématiques développées en psychologie culturelle et conceptualisées dans une perspective vygotskienne en termes de «zones de proche développement », « médiation », « étayage », qui guident leur activité de conception.

Le principe d'aide. Ce principe reprend, en les ajustant aux problèmes de formation, les propositions de Pinsky (1992) et Theureau \& Jeffroy (1994) qui préconisent la conception d'aides et non de prothèses. Les objets de la conception sont des artefacts qui ne se substituent pas aux acteurs au sens où ils réalisent la tâche à leur place, mais leur offrent des possibilités de gain de viabilité en étroite liaison avec leur activité usuelle. Ce principe est particulièrement exigeant s'agissant de formation, dans la mesure où ce qui est en jeu c'est l'apprentissage/développement et où l'aide porte sur ces processus et non la performance actuelle lors de la réalisation de la tâche (ce que Theureau $\&$ Jeffroy (1994) dénomment le registre fonctionnel de la construction versus celui de la détermination). Sa concrétisation nécessite de concilier les objectifs de performance et d'apprentissage/développement en raison de leur caractère souvent contradictoire: un apprentissage signifiant par exemple une baisse momentanée de performance dans une tâche professionnelle, le développement de l'activité un obstacle à son renouvellement par apprentissage... Ainsi par exemple, l'opérationnalisation de ce principe de conception d'aide se heurte à la question de l'opacité/transparence de ces artefacts. Un artefact efficace pour l'action est un artefact devenu « transparent » (Winograd \& Florès, 1986), tel par exemple que le clavier d'ordinateur qui une fois incorporé échappe à la conscience pré-réflexive de l'acteur et devient un instrument au sens de Rabardel (1995). S'agissant d'artefacts de formation, la question de la transparence constitue un dilemme: les artefacts enrichissant l'environnement des formés, et leur usage qui transforme leur couplage structurel doivent-ils ou non demeurer présents dans l'expérience des acteurs ou « devenir transparents »? Ce point n'est pas trivial comme l'indique l'analyse du recours à des artefacts particulier - les exemples - dans des séminaires de formation professionnelle (Perrin, soumis). Ceux-ci ont une fonction de concrétisation et de facilitation de l'assimilation d'énoncés abstraits, mais s'ils sont transparents pour les formés, ils remplissent de façon satisfaisante la fonction d'aide à la compréhension mais insatisfaisante celle d'aide à l'apprentissage/ développement.

Le principe de pari sur l'avenir et réduction d'ignorance. Ce principe concerne la contradiction inhérente au processus de conception ergonomique centrée sur l'activité, qui tient à ce que l'activité des acteurs n'est connaissable que lorsque ceux-ci sont effectivement confrontés aux environnements préalablement conçus (Pinsky, 1992; Theureau \& Jeffroy, 1994): la conception ne peut donc que procéder sur la base d'un pari relatif à ses effets sur l'activité des acteurs (dont on a vu qu'elle est ouverte et indéterminée), et une validation en cours de formation par réduction progressive de cette ignorance. Cela 
implique une démarche itérative et asymptotique, comme compromis alternant des phases de conception, d'analyse de l'activité des formés, d'ajustement des artefacts, d'analyse de l'activité des formés, etc. Cette ignorance est essentielle et constitutive de l'activité du concepteur: même si elle peut être diminuée a priori sur la base de connaissances et d'expériences diverses et a posteriori à partir de l'analyse de l'activité des acteurs confrontés aux versions successives des artefacts, elle est irréductible et contraint le concepteur à des engagements risqués. De sorte qu'on peut dire qu'il n'y a pas, en matière de conception, de recettes efficaces: le succès ne peut être programmé.

Le principe d'action à effet différé. Ce principe est classique mais fondamental en éducation et formation, où il s'agit d'accompagner une activité hic et nunc prometteuse d'un progrès utile ailleurs et plus tard. Ceci implique des hypothèses minimales relatives à l'état de l'environnement futur des formés. Il n'est notamment pas possible de considérer que l'environnement de travail et celui des pratiques sociales vont demeurer inchangés. Les recherches prospectives insistent par exemple sur l'accroissement prévisible de la flexibilité, l'invasion du numérique, l'organisation éphémère des entreprises et des environnements de travail, les communautés d'activité labiles et limitées à des projets ponctuels, le caractère paradoxal de la prescription (incomplétude, injonction de subjectivité...), etc. Ceci implique également des hypothèses (abordées au p.. 111-113) portant sur les transformations actuelles et immédiates du couplage structurel des formés, ou potentielles et différées, et leur stabilisation dans le temps. Ce principe incite à la vigilance notamment par rapport à l'un des traits de la conception centrée sur l'activité qui est son caractère conservateur: en se fondant sur l'activité actuelle, ces formations peuvent n'aboutir qu'à la reconduction de l'existant, et montrer leurs limites en matière d'innovation.

\section{Modélisation de l'activité cible et formation}

L'activité cible de la formation peut être le travail ou une quelconque pratique non laborieuse. Une connaissance précise de cette activité et notamment des composantes de son efficacité, est nécessaire pour concevoir des dispositifs et artefacts de formation.
Cette connaissance est acquise à partir d'une analyse systématique et a priori de l'activité d'acteurs dans les pratiques cibles. Cette étape d'analyse entraîne une rupture avec deux types de démarches usuelles. La première est celle de formateurs qui, possédant une expérience dans le travail ou la pratique cible, se fondent sur elle pour la conception des formations; la seconde propose des modélisations a priori des activités à partir d'une source d'information provenant des cahiers des charges ou plus largement de la prescription des pratiques. Ces démarches ayant montré leurs limites à de nombreuses occasions, ceci a incité à l'insertion d'une étape d'analyse de l'activité dans les procédures d'ingénierie de formation et au développement d'une didactique professionnelle centrée sur l'analyse de l'activité (Durand, sous presse b). La conception prend alors pour référence l'activité réelle des acteurs dans ce qu'elle a de typique (Durand, Meuwly-Bonte, \& Roublot, 2007; Durand, de Saint Georges, \& Meuwly-Bonte, 2005; Leblanc, Gombert, \& Durand, 2004; Ria, Leblanc, Serres, \& Durand, 2006).

Cette analyse préalable de l'activité cible en permet la modélisation qui devient un guide pour la conception. La modélisation repose sur l'identification des couplages types chez des praticiens expérimentés, ayant un caractère jugé critique par des formateurs ou des collectifs de pratiquants. Il s'agit de modèles synthétiques et dynamiques regroupant les constituants critiques de l'activité cible, qui permettent soit l'affinement et parfois la contestation des modèles usuels et des formations conçues en lien avec ceux-ci, soit la conception de formations innovantes dans le cas de pratiques jamais analysées ou émergentes.

Ainsi par exemple, des projets conduits en sport de haut niveau ont permis la conception d'artefacts intégrés à l'entraînement usuel donnant aux compétiteurs et entraîneurs l'accès à des dimensions préalablement ignorées (Durand, Hauw, Leblanc, Saury, \& Sève, 2004; Saury, 2008). En tennis de table, l'analyse de l'activité des meilleurs pongistes internationaux a permis une modélisation originale de cette activité (Sève, 2005) et la conception d'un plan de formation des joueurs et d'aides à l'entraînement innovants (Sève, Poizat, Saury, \& Durand, 2006). En trampoline, l'analyse a permis une critique du modèle de base de l'entraînement, qui résume cette activité 
à la production de formes corporelles principalement référée à la construction préalable de modèles mentaux, et à la reproduction en compétition de séquences motrices s'accompagnant d'une activité interprétative et décisionnelle réduite voire nulle, préalablement répétées et automatisées à l'entraînement. Or l'analyse de l'activité d'athlètes de niveau international a mis en évidence des traits incompatibles avec ce modèle, notamment: la non reproduction en compétition des figures répétées à l'entraînement, des prises de décisions et interprétations immédiates et complexes, des ajustements à des perturbations non anticipées dans des durées extrêmement brèves, une alternance subtile d'engagements visant soit à anticiper sur les événements soit à "laisser aller », etc. (Hauw, Berthelot, \& Durand, 2003; Hauw \& Durand, 2004, 2005). Ces résultats ont étayé un modèle de cette activité comme composée de deux séquences au cours desquelles les possibilités d'action diffèrent: celle du travail dans la toile correspondant à l'impulsion, et celle du travail aérien pendant le vol (Hauw, 2008). L'amélioration des performances dépend de l'utilisation par les athlètes des « possibles pour l'action » dans cette alternance. Ce modèle sommairement présenté ici, a guidé la conception de dispositifs d'aides à l'entraînement (Hauw, Berthelot, \& Durand, 2003; Hauw \& Durand, 2007).

L'étape d'analyse de l'activité n'est pas toujours un préalable indispensable à la modélisation dans la mesure où certaines pratiques ont déjà fait l'objet de recherches que le formateur peut exploiter: c'est le cas par exemple pour le travail des enseignants, qui est sans doute celui ayant été le plus étudié, et pour lequel les projets de formation peuvent démarrer sur la base d'une veille documentaire et une recension des recherches déjà publiées. Cependant, toutes les recherches ne sont pas exploitables si notamment leurs épistémologies ne recouvrent pas suffisamment les postulats de notre propre approche (rendre compte de l'activité en contexte naturel, hypothèse d'une totalité signifiante et insécable, présuppoés d'auto-organisation d'ouverture et indétermination, etc.). De sorte qu'en raison d'un manque d'analyses disponibles ou d'analyses raisonnablement compatibles, des analyses spécifiques sont souvent nécessaires. Celles-ci adoptent les démarches décrites dans le § 1.3, en tenant compte des conditions et notamment des ressources disponibles pour conduire cette analyse et cette modélisation.
La modélisation de l'activité experte ou de l'activité d'acteurs expérimentés a constitué une première base pour la conception de ces situations de formation. Cette pratique s'est rapidement développée pour diverses raisons (Durand, sous presse $a$ et $b$ ), notamment parce que les professionnels débutants rencontrent des problèmes particuliers que les expérimentés ont résolus et oubliés depuis longtemps; ces problèmes se trouvant masqués dans l'analyse de l'activité expérimentée ils ne sont pas intégrés dans la conception des formations. Ceci les rend moins efficaces parce que basées sur une connaissance partielle de l'activité cible et parce que les débutants sont moins réceptifs aux problématiques d'action des professionnels expérimentés. Ainsi l'activité des enseignants débutants a pu être modélisée à partir de trois expériences types: indétermination, contradiction et exploration (Ria, Sève, Durand, \& Bertone, 2004), qui la distingue nettement des modèles d'activité des enseignants expérimentés et a servi à la conception de formations destinées à accompagner « l'entrée dans le métier » de ces débutants (Leblanc, Ria, Dieumegard, Serres \& Durand, 2008; Ria, 2006; Ria et al., 2006). Par ailleurs, le repérage de ces spécificités des débutants a conduit à prendre en compte leurs parcours de formation et à modéliser leurs trajectoires professionnelles et ses transformations dans le temps (cf. p.. 114-117), c'est-à-dire non seulement leur activité professionnelle mais l'apprentissage/développement de celle-ci (Durand, 2007).

\section{Concevoir des espaces d'actions encouragées}

L'activité s'accompagne toujours potentiellement d'apprentissage/développement. Il n'y a donc pas lieu d'établir une distinction de nature entre un accomplissement productif ou laborieux, et l'activité spécifique d'apprentissage/développement. Cependant, certains environnements sont propices à des performances ici et maintenant, et d'autres à l'apprentissage/développement promettant des accomplissements futurs plus performants, sûrs, économiques, créatifs, inventifs, efficaces... On sait qu'il ne suffit pas de placer les formés en contact avec les aspects critiques de l'activité professionnelle préalablement modélisée pour les former; et c'est pourquoi le formateur conçoit des environnements d'apprentissage/développement conformes à ces modélisations de l'activité cible, mais dotés de propriétés particulières. Nous avons 
dénommés ces environnements d'apprentissage/développement des espaces d'actions encouragées (EAE) (Durand, sous presse a).

Les EAE sont des « dérivations » de la réalité ou des transformations des environnements de l'activité cible, qui portent sur une pluralité d'aspects: euphémisation des conséquences de l'action; critères de viabilité artificiellement institués; réduction de contraintes et simplification, ou à l'inverse complexification; rupture de temporalité (ralentissement ou accélération); focalisation sur des aspects particuliers de la pratique et mise en évidence des informations disponibles; encouragement et sollicitations d'émotions; délestage ou suppression des composantes subalternes des tâches qui sont momentanément isolées; incitations diverses, démonstrations, imitations, explications et enseignement ostensible; redoublement des expériences avec l'énoncé des règles d'action; alimentation de controverses et débats; mutualisation des offres pour agir; ajout de situations d'analyse des pratiques, d'environnements de simulation plus ou moins proches des pratiques cibles; procédures d'accompagnement, de tutorat, de coaching, d'étayage, etc. Dans le cadre théorique sémio-logique, l'objet de la formation est le couplage structurel des acteurs et sa transformation orientée, ce qui implique de concevoir des formations normatives mais non prescriptives. Elles sont normatives au sens où elles s'appuient sur une séparation entre les actions attendues, souhaitées et encouragées et celles qui sont "découragées » (nonobstant une place laissée la création et à l'inattendu dans le processus d'apprentissage/développement). Elles sont non prescriptives au sens où l'asymétrie du couplage structurel fait que c'est l'acteur qui définit ce qui, de son environnement, est significatif/pertinent pour lui: ce n'est donc pas l'environnement du formé - dont font partie le formateur et le dispositif de formation - qui spécifie son activité.

La notion EAE est empruntée notamment à Reed (1993; Reed \& Bril, 1996) moyennant un certain nombre de transformations (cf. Recopé, 2001). Les EAE sont des précurseurs prometteurs d'actions et d'expériences, supposés induire un apprentissage/ développement (Durand, sous presse a et b; Durand, de Saint Georges, \& Meuwly-Bonte, 2006; Durand, Meuwly-Bonte, \& Roublot, 2007 ; Leblanc, Durand, Saury, \& Theureau, 2001 ; Leblanc, Gombert, \&
Durand, 2004; Leblanc, Saury, \& Durand, 2003). Ces précurseurs évalués comme « prometteurs », concrétisent l'intention de perturber le couplage structurel des formés par un agencement de leur environnement pour déclencher des transformations, et sélectionner celles évaluées comme désirables. L'efficacité des EAE dépend de l'adéquation entre la structure d'attente des formés à l'instant $t$ (qui spécifie leur réceptivité aux perturbations/interventions formatrices), et le projet du formateur (qui cherche à ce que les appels à transformation que constituent ses interventions soient reçus et actualisés).

Les EAE actualisent la recherche par le formateur d'une influence sur l'activité du formé : ils encouragent certaines actions en les rendant possibles, en les facilitant, valorisant, validant, « offrant » (on parle alors d'affordance); ils créent des décalages ou des contradictions; ils découragent d'autres actions en spécifiant des impossibles ou des interdits. Les transformations du couplage structurel des formés et de leur environnement ne pouvant être ni instruites, ni complètement spécifiées a priori, la formation et sa conception sont nécessairement interactives au sens où l'environnement de formation ainsi conçu dépend de la capacité des formateurs à construire avec le (ou les) formé(s) un domaine consensuel (Maturana \& Varela, 1994), c'est-à-dire des opportunités d'articulations authentiques et non ambiguës de leurs couplages structurels respectifs. Ce domaine consensuel, jamais totalement pré-donné, est rendu plus probable par le partage de types entre le formateur et les formés et un ajustement asymptotique des significations co-construites en situation.

Les EAE résultent de la correspondance entre trois transformations: celles de l'environnement proche des formés sous l'effet des actions du formateur, celles du couplage structurel des formés avec leur environnement, et celle du couplage structurel $\mathrm{du}$ formateur sous l'effet de la transformation de son environnement consécutif aux effets observés chez les formés. Ces transformations et leurs correspondances sont à triple inconnues: le première concerne ce qui perturbe effectivement le couplage des formés et leur environnement; la deuxième l'orientation de leur dérive structurelle qui résulte d'un engagement continu et soutenu par le formateur de manière à favoriser ces trajectoires conceptualisées comme des dérives chaotiques; la troisième ce qui perturbe effec- 
tivement le formateur parmi l'ensemble des transformations qui se déroulent dans son environnement. Cela implique de prendre des distances avec une vision exclusivement planificatrice et programmatrice de la formation, et de penser les EAE comme des exploitations des potentiels des situations (Jullien, 1996), rompant ainsi avec une image de l'efficacité en termes d'anticipation et de planification exhaustives.

Enfin, cette approche conduit lors de la conception à une prise de distance avec la métaphore ${ }^{3}$ du contenant et du contenu qui est structurante de dispositifs de formation non tenus par le postulat d'enaction. Cette métaphore fait paraître évidente et naturelle la séparation entre des processus dynamiques et ce sur quoi opèrent ces processus qui est stable et invariant. Elle s'étend à des différences également prises pour allant de soi telles que " produit versus processus», " didactique versus pédagogie », " analyse du travail versus ingénierie de formation », " nature des savoirs versus objet des savoirs », etc. Les formations sont dans notre perspective conçues à partir du concept de «paire étoile » (Theureau, 2006; Varela, 1980), c'est-à-dire de la liaison entre un objet et le processus conduisant à cet objet (ceci/processus conduisant à ceci). Elles sont donc toujours auto-référentielles: l'activité étant à la fois contenu et processus et l'idée que l'activité soit l'objet de la formation, c'est-à-dire happé par un processus d'une autre nature, devient problématique. En effet si l'on conserve le caractère dynamique de toute pratique pour ne pas la réduire à des entités discrètes et statiques telles que les savoirs ou les représentations, et pour éviter la difficulté liée au passage depuis ces entités discrètes statiques vers le processus d'action, on aboutit à mettre en discussion la distinction entre processus et produit. Cette distinction renvoie notamment au schéma aristotélicien de l'hylémorphisme, c'est-à-dire l'idée que la formation donne une forme à une matière par nature hétérogène. Or si l'objet de la formation n'est pas un ensemble d'entités discrètes telles que les savoirs ou les représentations, mais un processus ou un dynamisme, et si celle-ci est elle-même un processus ou un dynamisme, on est conduit à la conceptualiser non pas comme une relation de saisie d'un objet par des processus qui peuvent à leur tour devenir des objets, mais comme une synthèse dynamique et complexe d'activité ou d'interactivité (Durand sous presse a; Fristalon \& Durand, sous presse).

\section{Favoriser une activité à caractère fictionnel en vue de l'apprentissage/développement}

Le formateur vise une amélioration de l'efficacité de l'activité des formés dans son environnement de travail ou de pratique cible, en proposant à ces formés des environnements particuliers au sein desquels leur activité diffère de l'activité cible. Cet écart est à la fois prometteur d'apprentissage/développement, et handicapant. Il est prometteur dans la mesure où il permet une marge avec l'activité cible qui ouvre la possibilité d'apprentissage/développement; il est handicapant car il rend problématique la remise en situation des acquisitions dans l'environnement cible. C'est pourquoi la conception d'EAE vise simultanément à établir une distance avec la pratique cible, mais aussi à minimiser cette distance. Les activités peuvent être distinctes à des degrés divers en fonction des degrés de ressemblance entre les environnements de formation et de travail. L'hypothèse sous laquelle sont conçus les environnements de formation est qu'une sorte de jeu doit être établie entre l'accomplissement cible réel et l'accomplissement en environnement d'apprentissage/développement, créant ainsi un débrayage (ou espace de transition si l'on adopte comme Pastré (2007) le concept de Winnicot) dont les propriétés constituent des conditions de l'apprentissage/développement de l'activité de travail.

C'est pourquoi nous avançons l'hypothèse que l'apprentissage/développement implique une « expérience fictionnelle ». Les formés déploient une activité (en lien avec les degrés de similitude entre environnements d'activité cible et de formation) analogue à celle que Shaeffer (1999) décrit comme une «production fictionnelle». Celle-ci repose sur un processus «d'immersion mimétique " dans l'environnement proposé par le formateur, un engagement marqué par une « feintise ludique partagée » et une "modélisation métaphorique de l'expérience » dans cet environnement ${ }^{4}$. Cette hypothèse n'assimile pas l'apprentissage/développement à une activité de production ou réception de fiction (théâtrale, cinématographique, romanesque...) mais avance qu'il y a nécessairement une composante fictionnelle active dans une formation.

L'immersion mimétique est le processus par lequel des expériences mimétiques sont réalisées à partir de 
leurres, qui permettent de « compter quelque chose pour autre chose ». C'est le cas des pratiques artistiques lorsqu'au théâtre l'action fictive d'un comédien est à l'origine d'émotions et interprétations de la part du spectateur du même ordre que celles observées face à la même action non jouée. Cette composante nous paraît toujours présente dans les dispositifs de formation dont les dimensions de leurre par rapport à l'environnement cible sont plus ou moins saillantes. Ceci est particulièrement évident dans les environnements dits de simulation qui sont des mimes des environnements cibles (Pastré, 2005 par exemple) et où ce qui est espéré des acteurs en formation est un «faire comme si ». Mais c'est aussi le cas dans des formations qui sollicitent de la part des formés une «mise en situation dynamique » par le visionnement de traces de leur activité ou de celles d'autres professionnels du même métier, ou lors de formations basées sur l'analyse de pratiques, ou encore de modèles de coaching ou d'entraînement (Durand, sous presse a; Leblanc et al., 2008; Ria et al., 2006). Dans tous ces cas, l'engagement des acteurs repose sur des ancrages non authentiques à des degrés divers mais ayant néanmoins quelque chose à voir avec des composantes des environnements cibles - ce quelque chose étant concrétisé par différentes sortes de leurres -. Il est attendu des formés qu'ils s'immergent dans des expériences en relation avec des ancrages différant peu ou prou des ancrages « réels » de l'activité cible. Les environnements de formation sont donc dans un rapport mimétique avec les environnements des pratiques cibles dans la mesure où les « leurres » conçus par le formateur sont « mis à la place » des ancrages réels du travail effectif.

La feintise ludique partagée se déploie, au fond, dans un sens inversé par rapport au processus d'immersion mimétique: elle implique de la part de l'acteur de distinguer sa situation actuelle de la situation cible sur la base d'un partage avec le formateur/concepteur de certains codes ou marqueurs. Autrement dit, les acteurs engagés dans une formation « font comme si » et, malgré l'immersion totale décrite précédemment, ne doivent pas prendre l'environnement d'apprentissage/développement pour l'environnement cible. Il s'agit pour eux de se déprendre de cette immersion dans le moment même où ils sont immergés. Cela est rendu plus ou moins facile en fonction des degrés de distance et d'explicitation de cette distance entre les environnements. L'environnement de formation doit
« s'annoncer », c'est-à-dire définir et délimiter l'espace de jeu à l'intérieur duquel opère le simulacre, ce qui permet que les environnements et leurs ancrages ne soient pas assimilés et interprétés comme réels. Cette feinte est toujours partagée entre le formateur et les formés (ce qui n'empêche pas les ambiguités et les quiproquos).

Ce débrayage entre deux ordres d'activité - le réel et le « comme si »-, caractérise un « jeu » entre réalité et environnement d'apprentissage/développement et confère une dimension de ludisme que nous pensons inhérente à toute expérience de formation. La conception d'environnements de formation dose et oriente potentiellement ce jeu, depuis les environnements les plus évidemment ludiques et de simulacres tels que les dispositifs dont le degré de proximité avec la réalité est troublant - voir par exemple la mesure «OuiStart, entreprise de pratique commerciale » proposée par l'État de Genève dans le cadre d'un programme d'entraînement de personnes en difficultés de ré-insertion dans le monde du travail, dans une entreprise virtuelle mais dont le fonctionnement est semblable à celui d'une entreprise réelle $e^{5}$, en passant par les environnements numériques de formation simulant des tâches de travail de façon schématique et destinés à solliciter pour les développer les composantes critiques de l'activité professionnelle - cf. l'exemple prototypique d'environnement de formation à la taille de la vigne conçu par Caens-Martin (1999) -, jusqu'aux environnements en apparence très éloignés des environnements de référence. Les environnements de simulation permettent à la fois des « débrayages » de l'activité par rapport aux pratiques cibles, et des marquages conventionnels (et partagés) de la présence d'une dimension ludique et de simulacre. Ce qui différencie ces environnements de formation tient à: la virtualité des actions notamment dans des environnements numériques, l'euphémisation des conséquences par déconnection avec les processus de production réels, la possibilité de recommencer, la tolérance accrue aux erreurs, l'augmentation de la quantité, au degré d'explicitation et de l'évidence des significations des informations relatives aux « conséquences de l'action », etc.

Le processus de modélisation métaphorique ${ }^{6}$ désigne les processus par lesquels les expériences liées à l'immersion mimétique et la feintise ludique 
peuvent être typicalisées et prendre la valeur de types ou prototypes qui permettent des extensions de la signification de ces expériences. L'extension s'opère sur la base d'engagements métaphoriques où un couplage vaut pour un autre couplage. Ces métaphores n'aboutissent pas à des énoncés ou des propositions généralisables ayant statut de savoirs explicites, mais on peut cependant parler à la suite de Schaeffer de modélisation: l'expérience ou le couplage sont modélisés au sens où des éléments de l'expérience dans les environnements de formation sont réunis en des ensembles cohérents et prégnants tels des « gestalts expérientielles » (Lakoff \& Johnson, 1980) et cette modélisation/métaphorisation constitue une condition de l'apprentissage (les nouveautés sont absorbées dans un premier temps par assimilation à du déjà vécu) et du développement (les métaphores permettent des pontages et une extension des couplages par une assimilation des environnements nouveaux aux anciens). La relation de similarité qui sous-tend la réactivation d'une expérience passée à partir de leurres, ne relève pas d'un isomorphisme de surface (encore que cela soit parfois possible), mais porte sur des aspects profonds d'organisation et de signification des expériences pour les formés (et qui, pour cette raison, peuvent échapper au formateur). Les types et métaphores sont à la base de ces extensions et extractions de signification à partir des environnements de formation dont l'efficacité en termes de formation est partiellement reliée à leur capacité à rendre possible ces processus de métaphorisation active.

\section{La formation individuelle à des activités collectives}

Dans une perspective enactive, l'activité collective est analysée sans présupposer une représentation ou un référentiel partagés préalables entre acteurs: le niveau collectif de structuration et de signification de l'activité est conceptualisé comme émergeant à partir des activités individuelles de chaque acteur et de leurs articulations locales (Durand, sous presse c; Theureau, 2006). Le caractère collectif de l'activité résulte d'un processus de totalisation à partir des articulations locales des activités individuelles, c'està-dire des ancrages locaux à la fois précis et ouverts, spécifiés par le fait qu'un aspect de l'activité d'un acteur « fait signe » pour un autre acteur et perturbe son activité. Ces articulations locales sont à l'origine d'une transformation de la structure et de la signification de l'activité de chaque acteur (Theureau, 2006). Ces articulations font émerger un niveau de structuration irréductible à celui des activités individuelles, qui les contraint en retour et leur confère une structuration et une signification nouvelles et étendues, plus ou moins stables. Ces formes collectives résultent aussi des éléments culturels partagés entre acteurs, les rendant ou faisant paraître naturelles, évidentes et non ambiguës.

Diverses analyses d'activité collective ont été réalisées dans le cadre de ce programme: entre enseignant et élèves dans une classe (Veyrunes, Durny, Flavier, \& Durand, 2005, 2007, sous presse), entre automobilistes à un carrefour (Durand, sous presse c), entre patient, infirmières et médecins au sein d'un service d'urgence à l'hôpital (Fristalon et Durand, 2008). Ces analyses montrent notamment que ces configurations n'impliquent pas nécessairement une réciprocité, et que même en cas de réciprocité, il est fréquent que les significations propres à chaque acteur ne soient pas totalement convergentes ou compatibles avec celles des autres acteurs. Ces articulations se coordonnent et rendent possibles l'actualisation de tout ou partie des composantes de l'expérience des acteurs impliqués. Cette profusion organisée d'activité aux significations multiples réalise une configuration irréductible aux activités individuelles et à leurs articulations deux à deux dont elle procède. Cette configuration a une structure et une signification qui englobent et reconfigurent les significations et structurations locales. Les couplages individuels sont fluctuants et basés sur une opacité réciproque et fondatrice de chacun pour l'autre et les degrés de spécification et d'indétermination de ces articulations varient selon les critères de viabilité des couplages: dans certains cas, une absence d'ambiguité est un critère de viabilité (lors d'un dialogue entre contrôleur aérien et pilote de ligne par exemple) et dans d'autres une dose élevée d'ouverture et d'indétermination est requise afin que des aspects explicites et clandestins ou masqués des activités individuelles puissent se réaliser (c'est le cas des interactions entre infirmières et patients par exemple).

Ces analyses pointent sur trois questions de conception formation principales liées à l'adoption d'un modèle interactif et auto-référencé de l'activité. 
La première est que ces analyses conduisent à une mise en question des modèles usuels adoptés en formation, basés sur un clivage de l'activité collective en deux composantes, l'une « technique * l'autre "relationnelle ». Cette dichotomie suppose qu'il n'y a pas de technique dans «le relationnel " ni de relation dans « le technique ». La composante technique est comprise comme une séquence d'actions efficace, codifiée, mécanique, déshumanisée ou non humanisée, et transmissible en tant que coordination d'actions pertinentes. La composante relationnelle est comprise comme relevant de processus tels qu'émotions, affects, sentiments propres aux dimensions conscientes et inconscientes de la rencontre avec l'autre, et exprimant une relation intersubjective non modélisable, non déterminée, non transmissible et acquise sur le tas. Cette dichotomie a pour conséquence soit d'évacuer de la formation cette dimension "relationnelle * en la reléguant dans l'informel, soit de l'insérer dans une "formation psychologique » générale déconnectée de la composante technique, soit de l'intégrer dans des formations non transmissives appuyées sur la réflexivité des acteurs et l'analyse de leurs pratiques. Nos analyses soulignent le caractère indissociable de ces deux composantes dont la séparation néglige le caractère " anthropologiquement constitutif de la technique » (Stiegler, 1994): pas d'humanisation ni d'humanité sans technique, et plus spécifiquement, pas de soins infirmiers, d'interactions professeur - élèves en classe ou de franchissement sûr d'un carrefour en automobile sans technique et sans relation. La conséquence en formation et de concevoir des formations sollicitant et préservant simultanément ces deux aspects.

La deuxième est liée à l'adoption à la suite de ces analyses, d'un modèle distribué du travail, c'està-dire d'une conceptualisation en termes de flux collectif d'activité. Ceci conduit à prendre au sérieux les niveaux de signification et de structuration de l'activité autres que ceux spécifiques à chaque acteur. Il s'agit d'accompagner la construction de capacités individuelles à agir collectivement de façon efficace, sans présupposer un niveau collectif préalable d'activité: l'efficacité dépend à la fois de la pertinence des activités individuelles et de celle de leurs articulations. Ceci caractérise une efficacité distribuée et émergente dont l'analyse conduit à deux orientations en formation: la première est l'objectif de déclenche- ment et d'accompagnement d'une prise de conscience par les formés des niveaux d'organisation de l'activité collective globale qui échappe usuellement à leur conscience et leur réflexivité, la deuxième est l'objectif de contribuer à la constitution de configurations viables et relativement stables (Durand, sous presse c), émanant de la participation d'acteurs à une entreprise commune et la constitution d'une culture partagée entre des acteurs n'ayant pas nécessairement le même travail à accomplir mais engagés dans des activités coordonnées et interdépendantes.

La troisième concerne le fait que le travail de formation suppose des articulations viables entre formateur et formés, et les questions énoncées cidessus relatives à ces pratiques collectives valent pour le travail formatif et les interactions formateur - formés. Celles-ci sont donc des articulations à visée formative d'activités individuelles qui concernent des articulations d'activités professionnelles individuelles. On se trouve alors dans une sorte de double boucle auto-référencée d'articulations d'activités individuelles. Ceci conduit à une mise en question (déjà pointé dans le § 2.3) de la distinction habituellement prise comme allant de soi entre contenu de formation (les soins infirmiers, l'enseignement, la conduite automobile) et le processus de formation (les interactions de formation).

\section{L'empan d'activité encouragée: de la situation de l'action aux dispositions à agir}

Le formateur intervient sur l'activité située des formés, alors que celle-ci ne l'intéresse pas directement puisqu'il cherche un effet sur une activité plus ou moins ressemblante, qui sera déployée ailleurs et dans le futur. Il cherche à générer des transformations viables à court, moyen et long termes dont, au fond, il ne connaît jamais le degré de recoupement ou de superposition: des transformations ici et maintenant peuvent n'être pas suivies de transformations à moyen et long terme, et inversement. Alors que sa seule fenêtre d'intervention est l'accomplissement de l'activité située, il lui faut supposer des processus assurant un pont entre l'activité au sein des dispositifs de formation et l'activité future dans la pratique cible, c'est-à-dire des processus ou des objets qui aient une pérennité pour assurer ce pontage, et une généralité pour couvrir des actions dans des environnements 
différents de ceux où s'est produit leur apprentissage et initiée leur développement.

Ceci nous conduit, à partir de divers auteurs (notamment Bourdieu, 1980; Bourdieu, 1998 ; Peirce, 1995; Varela, 1996), à évoquer une notion centrale de tout projet de formation : la « disposition ». Cette notion articule dans la conceptualisation et la conception de l'activité : le social et l'individuel, le possible l'actuel et le virtuel, le déjà là et l'émergent, le réglé, le régulier et l'inédit, la conservation et l'invention, etc. Nous parlons plutôt de « dispositions à agir » pour marquer l'ancrage dans une approche de l'activité, sans cependant restreindre ces dispositions à des actions pratiques stricto sensu : les dispositions à agir concernent aussi les composantes interprétatives, émotionnelles, cognitives, perceptives, intentionnelles... de l'activité. L'idée générale est que toute action structurée et signifiante peut être comprise comme la traduction ou l'actualisation d'une disposition à agir qui en assure le caractère organisé, signifiant et réglé Dans une approche enactive, les dispositions à agir sont conceptualisées comme des possibles dont l'actualisation en contexte n'est ni automatique ni totalement prescriptive des actions qui émergent d'interactions locales entre objets ou processus. Ce sont la régularité, la signification, la reproductibilité des actions qui sont désignées de dispositions à agir, et non une entité autre que l'action et pré-existante qui commanderait, prescrirait, déterminerait l'accomplissement situé. Au plan théorique cette notion permet de circuler depuis les approches de l'action située vers les théories du sujet: les dispositions à agir sont les construits qui articulent l'idée d'émergence en contexte d'actions efficaces organisées et signifiantes, et de sujets présentant une certaine stabilité de comportements, c'est-à-dire une certaine régularité d'activité voire une identité tout court.

Sans entrer dans le détail des débats théoriques associés à ce concept, nous livrons dans les lignes qui suivent les éléments nécessaires à son exploitation lors de la conception d'artefacts formatifs. Ces éléments concernent deux aspects: d'une part les propriétés de l'activité que nous désignons par « dispositions à agir », et d'autre part les contributions des dispositions à agir à l'apprentissage/développement, à la participation à des collectifs et à la construction identitaire.

\section{Propriétés dispositionnelles de l'activité}

La notion de disposition à agir désigne un ensemble de propriétés de l'activité parmi lesquelles certaines sont critiques au regard des exigences de la conception de dispositifs de formation; ce sont: a) la régularité et la reproductibilité des actions, b) l'autonomie relative par rapport aux environnements, c) la relation de détermination potentielle avec l'action située, d) la propension à s'actualiser et e) le caractère acquis de certaines caractéristiques de l'action qui suppose un apprentissage.

L'activité présente des régularités en lien avec des ressemblances entre environnements d'action, de sorte que des attentes de reproduction jouent comme des « allants de soi »: si l'on a appris à faire de la bicyclette on s'attend à ce que, même après une interruption prolongée, on n'ait pas à ré-apprendre cette habileté ou au moins à ce que ce ré-apprentissage soit plus rapide et facile que le premier. Cette reproductibilité et cette régularité tiennent à des similitudes entre environnements (qui présentent des « airs de famille »), et à des engagements semblables de la part des acteurs. La notion de disposition à agir désigne ces régularités/reproductibilités au sens où elles expriment des possibilités de ressemblances.

L'autonomie relative à l'égard des environnements est d'une certaine manière le contrepoint de la propriété de régularité: elle désigne des écarts à cette régularité pensés comme non accidentels. La disposition à agir exprime une relative autonomie à l'égard des environnements, et consiste en un étiquetage de processus de « dé-situation » d'actions analogues réalisées dans des environnements différents, ou d'actions différentes dans des environnements analogues. Elle désigne donc à la fois le caractère ontologiquement situé de l'action et la possibilité d'un certain « débrayage » entre activité et environnement, qui permet une malléabilité du couplage structurel.

Les deux propriétés précédentes alimentent l'idée que les dispositions sont des potentiels d'action: elles ne sont pas mécaniquement actualisées dans la mesure où l'action ne se répète pas toujours à l'identique en dépit d'environnements apparemment similaires. Cette propriété est résumée par l'idée que les dispositions à agir peuvent ou pourraient être actualisées à chaque instant ou bien que l'action est une 
actualisation indéterminée de certains possibles. À chaque instant sont ouverts certains possibles, mais le champ des possibles est délimité en même temps qu'il est ouvert. Les dispositions à agir sont ainsi conceptualisées dans un cadre peircien articulant trois registres: priméité correspondant à une ouverture de possibles, secondéité correspondant au choc de la réalité et du «ici et maintenant ", et tiercéité correspondant à la règle et à la généralité (voir pour une analyse approfondie Theureau, 2006). Dans son accomplissement situé, l'activité articule et fonde ces trois registres qui dénotent le caractère indéterminé et ouvert de l'action, le processus d'actualisation de possibles issus des expériences antérieures, et la possibilité de la généralisation.

Les dispositions à agir désignent aussi une propension à l'actualisation. Elles caractérisent a) une tension anticipatrice vers un accomplissement particulier ou une action effective possible, b) secondairement une tendance à la régularité qui a pour conséquence un certain conservatisme: dans un environnement interprété comme semblable à d'autres où une action a déjà été réalisée et estimée suffisamment efficace, l'acteur tend à la re-produire, et c) une résistance corrélative au changement: il faut refréner la propension à l'actualisation pour agir « autrement ».

Les dispositions à agir désignent enfin un apprentissage/développement dont elles sont le produit: des actions préalablement réalisées par un acteur, dans un cadre de formation ou non, sont accomplies à nouveau plus tard et dans d'autres environnements. Cette traversée des environnements est considérée comme l'expression de types, c'est-à-dire le produit de la typicalisation d'une expérience significative, d'un échantillon d'expériences, ou de mises en relations entre expériences déjà typicalisées (Theureau, 2006). La typicalisaton assure une généralité à l'action située. Il ne s'agit pas d'une abstraction et décontextualisation, mais d'un processus par lequel une expérience prend valeur de type, et sert ensuite d'ancrage à des expériences ayant avec elle des éléments de ressemblance, constituant ainsi une sorte de "familles de couplages ».

\section{Formation et dispositions à agir}

Le second ensemble de traits à considérer en matière de formation est que les dispositions à agir sont simultanément tournées vers l'apprentissage/ développement, la socialisation/participation à des collectifs et la construction identitaire.

Les dispositions à agir désignent une forme de sédimentation de couplages antérieurs ou d'expériences passées. Comme telles elles relèvent de la problématique de la compétence telle que développée dans le champ de la formation, c'est-à-dire des constructions permettant à l'acteur d'acquérir des potentiels d'action de plus en plus efficaces qui intègrent toutes les dimensions de l'expérience. Cet apprentissage s'accompagne de la transformation des dispositions déjà là, et l'acteur est pris entre une tendance au conservatisme (il résiste à invalider son répertoire parce que celui-ci assure toujours une relative efficacité) et à l'innovation (il est, jusqu'à la crédulité, attiré par la nouveauté potentiellement efficace). Cette dynamique de la compétence articule, entre doute et confiance, deux composantes: le développement de celles déjà là (par différenciation, automatisation, extension, institution...) et l'acquisition de nouvelles (par transmission, imitation, intériorisation...).

Les dispositions à agir désignent aussi une sorte de pont entre les dimensions collectives et individuelles. Bourdieu (1979), reprenant une part de l'argumentation de Peirce à ce propos, a insisté sur la conceptualisation des habitus comme ensemble de dispositions, résultant des processus de socialisation (notamment primaire), et marquées par les appartenances sociales des acteurs: les dispositions désignent alors certains modes d'action collectivement valorisés, c'est-à-dire la hiérarchie agissante des modes de faire dans tel groupe social. Elles sont un ingrédient des processus de participation, distinction et domination sociales. La dynamique de la participation concerne l'évolution avec le temps des interactions d'un acteur qui pénètre dans une communauté. Cette participation implique le passage de outsider à insider et un double processus de désengagement/engagement. Les interactions avec les " déjà membres » prennent des formes évolutives: il s'agit d'interactions de tutelle ou d'étayage des anciens envers les débutants, qui deviennent plus rares avec le temps jusqu'à prendre la forme de négociations, débats ou controverses relatives à « la bonne pratique » au sein de la communauté. Les transformations de l'activité des nouveaux venus marquent un changement 
de leur place au sein de la communauté et de leurs interactions avec les autres participants. Ces interactions ont pour objet les valeurs et les types d'action propres à la communauté, que le nouveau venu intègre en les mobilisant. Un processus de légitimation est en jeu dans cette dynamique: a) légitimation des nouveaux venus qui par la conformation de leur activité gagnent une place plus centrale dans la communauté, b) légitimation des anciens en tant que gardiens de la bonne pratique du fait de l'acceptation de leurs jugements par les nouveaux venus, c) légitimation de la pratique en question et d) légitimation de la communauté (Goudeaux, Stroumza, \& Durand, 2008 , sous presse). Ces processus de légitimation sont eux aussi en tension entre deux quêtes interactives contradictoires: l'entretien et la conservation de la bonne pratique (c'est-à-dire la pratique convenable et convenant) et l'invention de nouvelles pratiques et la réforme de l'ancienne.

Enfin les dispositions à agir désignent la « matière identitaire $»$ : des sujets qui ne sont rien d'autre que leurs actes ont une consistance, une permanence, une redondance dans le temps en dépit de la diversité des environnements. La redondance et l'identité ne sont pas préalables à l'activité mais sont avec elle dans un rapport de couplage: l'activité de l'acteur si elle connaît des transformations permanentes présente aussi des régularités et des redondances qui peuvent être désignées comme des expressions identitaires d'un sujet et d'un environnement relativement stables. Au fil de ces transformations, le couplage structurel fait émerger des micro-identités et des micro-mondes (Varela, 1996) : le fait de marcher dans un environnement fait émerger un « micro-moi marcheur » et un « micro-monde marchable », qui deviennent un « micro-moi coureur » et un « micromonde courable » si une autre coordination sensorimotrice est actualisée. Les environnements sociaux actuels, qui se caractérisent par leur dynamisme et leur différenciation favorisent l'émergence de microidentités individuelles multiples qui font présenter les egos modernes comme morcelés, fragmentés, pluriels (Lahire, 1998). Ceci tient à ce que les dispositions s'organisent en réseaux ayant une spécificité qui explique les «inconsistances biographiques » globales des individus qui peuvent être très différents selon les environnements, mais autorisent aussi à parler d'unité et d'identité. Ces dynamiques peuvent être résumées par une tension entre deux orienta- tions: a) une recherche de "mêmeté » et de cohérence au plan individuel (être soi-même, être fidèle à soi-même, se sentir différent des autres et conforme à ce qu'on est) et au plan collectif (affirmer la spécificité de sa communauté, sa valeur et ses traits) versus b) une assimilation individuelle (être et se sentir comme les autres) et collective (affirmer son appartenance, démontrer qu'on est membre).

Il y a un intérêt, pour un formateur, à envisager conjointement ces trois dynamiques lors de la conception de formations. Les dispositions à agir nous paraissent être les construits intermédiaires assurant une cohérence entre tendances divergentes au sein des dynamiques de l'activité et de ses transformations, et les notions permettant de cadrer cette activité de conception.

\section{Conclusion}

Cette présentation laisse dans l'ombre un certain nombre de points liés aux relations de ce programme avec d'autres plus ou moins proches et à ses enjeux sociétaux. Cette brève conclusion se limite à lister quelques points essentiels.

Ce programme technologique entretient des relations d'interdépendance à l'intérieur de ce qu'on peut décrire comme une généalogie engendrée par l'approche générale et princeps du cours d'action (Theureau, 2004, 2006, sous presse) et constituée des programmes tenus par le même ensemble de présupposés et hypothèses mais portant sur des domaines de pratiques particuliers comme par exemple le programme de recherche en musicologie empirique (Donin \& Theureau, 2007; Theureau \& Donin, 2006), en analyse de la performance sportive (Hauw, 2008; Saury, 2008; Sève, 2005), le programme philosophique « cours d'action» (Theureau, sous presse). Ce programme technologique prétend contribuer à définir des objets originaux, et proposer des notions et dispositifs susceptibles d'être exploités dans les autres programmes de la «famille cours d'action ». Comme on l'a vu, ils traitent avec insistance de questions d'influence des couplages structurels, de construction de domaines cognitifs consensuels, d'articulation des couplages, d'interaction et d'activité collective, de navigation d'un espace social à l'autre, de pérennisation des transformations et du développement, de 
diversification et coordination des échelles de temps dans l'analyse de l'activité... qui sans être des objets centraux des autres programmes, sont susceptibles de les intéresser à un titre ou un autre. Les apports potentiels sont aussi d'ordre méthodologique avec des innovations portant par exemple sur le recours à des traitements quantitatifs de séries temporelles d'observations répétées, des tentatives de documentation de l'activité des acteurs dans des perspectives d'étude du cours de vie des acteurs (relatifs ou non à une pratique), la conception d'artefacts informatiques exploitables tant en recherche qu'en formation (Perrin, Theureau, Menu, \& Durand, soumis), etc.

Par ailleurs ce programme a pour ambition de contribuer à une réflexion élargie sur la formation en général, la professionnalisation et toutes les modalités d'intervention ciblées par des pratiques sociales. De ce fait il est partie prenante des débats éthiques relatifs notamment à la nature des relations humaines au sein des pratiques sociales et à la signification collective de la formation à ces pratiques: tricherie en sport de haut niveau, «sale boulot » dans les pratiques professionnelles, complaisance face à des pratiques de formation notoirement inefficaces, assujettissant des formés aux exigences de l'organisation du travail, exclusivité des objectifs de rentabilité... Cela rend nécessaire une vigilance quant à la contamination possible du champ de la formation par les valeurs issues des champs de pratique ciblées. Une affirmation des positions explicites relatives au type de sujet que les formations ont l'ambition d'accompagner est nécessaire (cf. Theureau (sous presse) pour une discussion approfondie de ces questions). Il s'agit pour les participants à ce programme de promouvoir un idéal d'acteur actif et en développement permanent, non réductible à son rôle social et sa tâche professionnelle, raisonnable et réflexif, non scindé entre raison et passion, autonome et responsable individuellement et collectivement. Ces ambitions sont exigeantes et parfois contradictoires avec la conduite même de ce programme technologique. Ainsi par exemple, du mouvement de "professionnalisation » qui concerne l'ensemble des pratiques sociales (et au premier chef les pratiques professionnelles), auquel ce programme contribue en reprenant les objectifs de rationalisation des procédures et règles des métiers et des pratiques, par une expression des expertises et une externalisation concomitante des fonctions de formation, de recrutement, voire d'évaluation des pratiques. Il existe en effet des possibilités non négligeables d'effets secondaires liés à la spécialisation, rationalisation et séparation de ces activités dans la mesure où elles induisent des risques de dévitalisation des pratiques sociales par une dissociation des fonctions de mémorisation, transmission et invention qui étaient, dans des organisations professionnelles passées, spontanément assurées par les pratiques elles-mêmes (Durand, 2006). Ainsi certains acteurs et groupes d'acteurs particulièrement dynamiques et autonomes, structurent leur travail de manière à assurer ces fonctions de façon consubstantielle à leur accomplissement laborieux, et cette intrication étroite avec le travail constitue pour eux, une condition de l'efficacité de la pratique et du développement des collectifs et des individus (Goudeaux, Stroumza $\&$ Durand, 2008, sous presse).

De plus, ce programme technologique s'oriente aujourd'hui vers un objectif nouveau de contribution à l'analyse des formes de relation entre travail et formation et à l'invention de formes nouvelles et émancipatrices (Durand, sous presse d). De nombreux processus mis en avant par les sociologues de la modernité et dans les rapports de prospective portant sur le travail et son organisation décrivent une évolution probable vers l'organisation des pratiques sociales en projets éphémères et contraints dans le temps, la constitution de groupes professionnels relevant non de métiers mais de communautés d'activité, la généralisation de l'intermittence et de la migration sociale, l'implantation de formations au contact du travail ou de la pratique cible, l'exigence de créativité et d'inventivité, la violence symbolique et pratique des procédures d'évaluation (critères peu explicites et changeants, conséquences disproportionnées), la légitimation et institution des pratiques par la procédure, l'invasion de toutes les pratiques par un système marchand, etc. Ces perspectives à peine esquissées ici questionnent les formations actuelles qui a) sont en général pensées en termes de métiers ou de pratiques établis, de collectifs pérennes, de référentiels de compétences stables, de structuration identitaire des individus prioritairement par le travail..., b) sont muettes quant aux évolutions des pratiques cibles ou affichent un angélisme magnifiant l'intelligence des situations et la créativité de l'agir en masquant les enjeux économico-politiques et de domination généralement liés à ces évolutions. 
Enfin, l'articulation de deux programmes de recherche empirique et technologique affirme aussi dans le champ des Sciences de l'éducation une position épistémologique particulière et qui ne va pas de soi (Durand, sous presse d). Un tel positionnement a) conduit à penser la technique et la technologie autrement que comme séparées et dans une relation de dépendance par rapport aux savoirs et à la science, b) incite à se préoccuper davantage de technologie et de technique que ce n'est actuellement le cas dans le domaine de l'éducation et de la formation, c) interroge sur la place et la signification à l'université de la discipline "Sciences de l'éducation » au sein de laquelle ce programme se développe, d) pose plus largement la question des relations entre programmes (de recherche et technologique) et disciplines universitaires. 


\section{NOTES}

1. Je remercie la rédaction de la revue Education et Didactique pour sa sollicitation de cet article, ainsi que I. Fristalon, A. Goudeaux, D. Hauw, S. Leblanc, M. Meuwly-Bonte, L. Ria, J. Saury, C. Sève, K. Stroumza, J. Theureau, P. Veyrunes pour leurs commentaires de sa première version.

2. Il arrive que des projets technologiques soient initiés par les membres de l'équipe eux-mêmes. Cela se produit lorsque ces membres sont également engagés dans les pratiques extra-universitaires où se réalise le projet, ou lorsque les projets concernent directement les pratiques universitaires.

3. Métaphore est entendu ici au sens de Lakoff \& Johnson (1980), c'est-à-dire une structure expérientielle qui agrège des cognitions, émotions, perceptions... et constitue un cadre d'interprétation du monde et d'action dans ce monde.

4. Ces processus sont présentés ici à partir des propositions de Schaeffer (1999) sur l'expérience fictionnelle, mais notre analyse emprunte aussi à Zaccai-Reyners (2005) qui a proposé de mettre en relation ces processus fictionnels et les processus de typification selon Schütz. Nous ne reprenons cependant pas point pour point les arguments de ces deux auteurs qui s'inscrivent dans une épistémologie cognitiviste.

5. http://www.ouistart.ch/accueil.htm

6. Nous ne reprenons pas ici la formulation de Schaeffer qui parle lui de «modélisation analogique » en s'appuyant sur l'hypothèse de processus d'abstraction et de représentation que nous contestons.

\section{RÉFÉRENCES}

Bourdieu, E. (1998). Savoir faire. Contribution à une théorie dispositionnelle de l'action. Paris: PUF.

Bourdieu, P. (1979). La distinction. Critique sociale du jugement. Paris: Minuit.

Bourdieu, P. (1980). Le sens pratique. Paris: Minuit.

Brocklesby, J., \& Mongers, J. (2006). The use of the concept of autopoiesis in the theory of viable systems. Systems Research and Behavioral Science, 22, 3-9.

Caens-Martin, S. (1999). Une approche de la structure conceptuelle d'une activité agricole: la taille de la vigne. Éducation Permanente, 139, 99-114.

Donin, N., \& Theureau, J. (2007) Theoretical and methodological issues related to long term creative cognition: The case of musical composition. Cognition, Technology \& Work, 9 (4), 233-251.

Durand, M. (2006). Activité(s) et formation. Genève: Carnets de la Section des Sciences de l'éducation.

Durand, M. (2007). Situations de l'action, dispositions à agir et trajectoires d'activité chez des enseignants débutants et des formateurs de terrain. Formation et Pratiques d'Enseignement en Questions, 6, 59-74.

Durand, M. (sous presse a). Favoriser l'apprentissage et le développement professionnels: la conception d'espaces d'actions encouragées visant la construction de dispositions à agir. In M. Durand \& L. Filliettaz (Eds.), La place du travail dans la formation des adultes. Paris: PUF.

Durand, M. (sous presse b). Analyse du travail dans une visée de formation: cadres théoriques, méthodes et conceptions. In J.-M. Barbier, E. Bourgeois, G. Chapelle et J. C. Ruano-Borbalan (Eds.), Encyclopédie de la formation. Paris: PUF.

Durand, M. (sous presse c). Concevoir des formations en articulation avec l'analyse de l'activité individuelle et collective: réflexion à partir de cas de conduite et d'inconduite automobile. In M. Durand \& F. Saussez (Eds). Entre l'individuel et le collectif: les potentialités d'une entrée par l'activité en formation. Bruxelles: De Boeck.

Durand, M. (sous presse d). Mutation des relations travail - formation et transformation des savoirs: une perspective enactive en éducation des adultes. Raisons Éducatives, 13. 
Durand, M., de Saint Georges, I., Meuwly-Bonte, M. (2005). Le curriculum en formation des adultes : argumentation pour une approche « orientée - activité ». Raisons Éducatives, 9, 185-202.

Durand, M., Hauw, D., Leblanc, S., Saury, J., \& Sève, C. (2004). Analyse de pratiques et entraînement en sport de haut niveau. Education Permanente, 161, 54-68.

Durand, M., Meuwly-Bonte, M., \& Roublot, F. (2007). Un programme de technologie de formation centré sur une approche auto-référencée de l'activité. Travail et Apprentissages, 1, 76-91.

Fristalon, I., \& Durand, M. (2008). Analyse du travail infirmier comme interactivité et formation professionnelle. Raisons Éducatives, 12, 275-297.

Giard, L. (1994). Introduction. L'impossible désir du rationnel. In I. Lakatos, Histoire et méthodologie des sciences (p.. V-XLIII). Paris: PUF.

Goudeaux, A., Stroumza, K., \& Durand, M. (2008). Communauté de pratique et configuration d'activité: la légitimation de la pratique chez les accessoiristes du Grand Théâtre de Genève. Pratiques de formation Analyses, 54, 67-78.

Goudeaux, A., Stroumza, K., \& Durand, M. (sous presse). Faire advenir des solidarités, compétences et identités dans les relations de travail: analyse des dimensions formatives de l'activité des accessoiristes de théâtre. Education et Sociétés.

Hauw, D. (2008). Une modélisation de l'activité acrobatique élite. Note de synthèse non publiée pour l'habilitation à diriger les recherches. Université de Montpellier I.

Hauw, D., Berthelot, C., \& Durand, M. (2003). Enhancing performance in elite athletes through situatedcognition analysis: Trampolinists'course of action during competition activity. International Journal of Sport Psychology, 34, 299-321.

Hauw, D., \& Durand, M. (2004). Elite athletes'differentiated action in trampolining: A qualitative and situated analysis using retrospective interviews. Perceptual and Motor Skills, 98, 1139-1152.

Hauw, D., \& Durand, M. (2005). How do elite athletes interact with the environment in competition? À situated analysis of trampolinists'activity. European Review of Applied Psychology, 55, 207-215.

Hauw, D., \& Durand, M. (2007). Situated analysis of elite trampolinists'problems in competition using retrospective interviews. The Journal of Sport Sciences, 25 (2), 173-183.

Jullien, F. (1996). Traité de l'efficacité. Paris: Grasset.

Lahire, B. (1998). L'homme pluriel. Les ressorts de l'action. Paris: Nathan.

Lakatos, I. (1994). Histoire et méthodologie des sciences. Paris: PUF.

Lakoff, G., \& Johnson, M. (1980). Metaphors we live by. Chicago : University of Chicago Press.

Le Boterf, G. (2003). L'ingénierie: concevoir des dispositifs dans des environnements complexes et évolutifs. Education Permanente, 157, 53-61.

Leblanc, S. Ria, L., Dieumegard, G., Serres, G., \& Durand, M. (2008). Concevoir des dispositifs de formation professionnelle des enseignants à partir de l'analyse de l'activité au travail et en formation, @ctivité(s), 5 (1), 58-78.

Leblanc, S., Durand, M., Saury, J., \& Theureau, J. (2001). Knowledge construction during multimedia user's action. Computers \& Education, 36, 59-82.

Leblanc, S., Gombert, P., \& Durand, M. (2004) (Eds.). Réfléchir les pratiques: sport, éducation, formation. Version CD Rom. Ecole Nationale de Voile \& IUFM de Montpellier.

Leblanc, S., Saury, J., \& Durand, M. (2003). Les interactions utilisateurs-environnement hypermédia en situation réelle de formation. Savoirs, 3, 55-74.

Maturana, H.R., \& Varela, F.J. (1994). L'arbre de la connaissance. Racines biologiques de la compréhension humaine. Paris: Addison-Wesley France. 
Pastré P. (2005) (Ed.). Apprendre par la simulation: de l'analyse du travail aux apprentissages professionnels. Toulouse: Octarès.

Pastré, P. (2004). Introduction. In R. Samurçay \& P. Pastré (Eds.), Recherches en didactique professionnelle (p.. 1-13). Toulouse: Octarès.

Pastré, P. (2007). La didactique professionnelle: origines, fondements, perspectives. Apprentissages et Travail, $1,9-21$.

Peirce, C.S. (1978). Écrits sur le signe. Paris: Seuil.

Peirce, C.S. (1995). L'habitude. In C. Chauviré, P. Thibaud, P \& C. Tiercelin (Eds.), Le raisonnement et la logique des choses (p.. 283-310). Paris: Les Éditions du Cerf.

Perrin, N. (soumis). Usage, appropriation et transparence des exemples en formation professionnelle initiale des enseignants. In D. Adé \& I. de Saint Georges (Eds.), Les objets dans la formation et l'apprentissage. Toulouse: Octarès.

Perrin, N., Theureau, J., Menu, J., \& Durand, M. (soumis). SIDE-CAR: un outil d'aide à l'analyse de l'activité selon le cadre théorique du cours d'action. @ctivité(s).

Piaget, J. (1974). La prise de conscience. Paris: PUF.

Pinsky, L. (1992). Concevoir pour l'action et la communication: essais d'ergonomie cognitive. Berne: Peter Lang.

Rabardel, P. (1995). Des hommes et des techniques: une approche cognitive des instruments contemporains. Paris: Armand Colin.

Recopé, M. (2001). L’apprentissage. Paris: Éditions EP.S.

Reed, E. (1993). The intention to use a specific affordance: A conceptual framework for psychology and context. In R.H. Wosniack \& K.W. Fisher (Eds.), Development in context: Acting and thinking in specific environments (p.. 45-76). Hillsdale: Erlbaum.

Reed, E., \& Bril B. (1996). The primacy of action in development. À commentary of N. Bernstein. In M. Latash $\&$ M. Turvey (Eds.), Dexterity and its development (p.. 431-451). Hillsdale, NJ: Erlbaum.

Ria, L. (2007). L'entrée dans le métier des enseignants du second degré: un programme de recherche centré sur l'analyse de l'activité. Note de synthèse non publiée pour l'Habilitation à Diriger les Recherches. Université de Clermont-Ferrand

Ria, L., Leblanc, S., Serres, G., \& Durand, M. (2006). Recherche et formation en « analyse de pratiques »: un exemple d'articulation. Recherche et Formation, 51, 43-56.

Ria, L., Sève, C. Durand, M., \& Bertone, B. (2004). Indétermination, contradiction et exploration: trois expériences typiques des enseignants débutants en Education Physique. Revue des Sciences de l'Education, 3, 535-554.

Saury, J. (2008). La coopération dans les situations d'intervention, de performance et d'apprentissage en contexte sportif. Note de synthèse non publiée pour l'habilitation à diriger les recherches. Université de Nantes.

Schaeffer, J.M. (1999). Pourquoi la fiction? Paris: Seuil.

Sève, C. (2005). Une modélisation de l'activité en match de pongistes de haut niveau. Note de synthèse non publiée pour l'Habilitation à Diriger les Recherches. Université de Rouen.

Sève, C., Poizat, G., Saury, J., \& Durand, M. (2006). Un programme de recherche articulant analyse de l'activité en situation et conception d'aides à la performance: un exemple en entraînement sportif de haut niveau. @ ctivité(s), 3 (2), 46-64.

Simondon, G. (1989). L'individuation psychique et collective. Paris: Aubier.

Stiegler, B. (1994). La Technique et le temps. Tome I: La faute d'Epiméthée. Paris: Galilée.

Suchman, L. (1987). Plans and situated actions: The problem of human-machine communication. Cambridge: Cambridge University Press.

Theureau, J. (2004). Le cours d'action. Méthode élémentaire. Toulouse: Octarès. 
Theureau, J. (2006). Le cours d'action. Méthode développée. Toulouse: Octarès.

Theureau, J. (sous presse). Le cours d'action: Méthode réfléchie. Toulouse: Octarès.

Theureau, J., \& Donin N. (2006). Comprendre une activité de composition musicale. In J.M. Barbier \& M. Durand. (Eds.) Les rapports sujets-activités-environnements (p.. 221-251). Paris: PUF.

Theureau, J., \& Jeffroy, F. et al. (1994). Ergonomie des situations informatisées: la conception centrée sur le cours d'action. Toulouse: Octarès.

Tsuchiya, Y. (2006). Autopoietic viable system model. Systems Research and Behavioral Science, 24, 19-35.

Varela, F.J. (1980). Principles of biological autonomy. New York: Elsevier.

Varela, F.J. (1989). Autonomie et connaissance. Paris: Seuil.

Varela, F.J. (1996). Quel savoir pour l'éthique? Paris: La Découverte.

Varela, F.J., \& Shear, J. (1999). First person methodologies: What, why, how? Journal of Consciousness Studies, $6(2-3), 1-14$.

Veyrunes, P., Durny, A., Flavier, E., \& Durand M. (2005). L'articulation de l'activité de l'enseignant et des élèves pour résoudre un problème de mathématiques à l'école primaire: une étude de cas. Revue des Sciences de l'Éducation, 31-2, 471-489.

Veyrunes, P., Gal-Petitfaux, N., \& Durand, M. (2007). La lecture orale au cycle 2: configuration et viabilité de l'activité collective dans la classe. Repères, 36, 59-76.

Veyrunes, P., Gal-Petitfaux, N., \& Durand, M. (sous presse). Configurations of activity: From the coupling of individual actions to the emergence of collective activity. Research Papers in Education.

Von Uexküll, J. (1965). Mondes animaux et mondes humains. Paris: Gonthier.

Winograd, T., \& Florès, F. (1986). Understanding computers and cognition: A new foundation for design. Norwood, N.J. : Ablex.

Zaccaï-Reyners, N. (2005). Fiction et typification. Methodos, 5. 